\title{
Evaluation Reliability of the Control Building
}

\author{
Materials and Products for Stability
}

\section{Technological Processes of Production}

\author{
V. I. Loganina \\ Street Titov, 28. 440028 Penza, Russia
}

\begin{abstract}
Copyright (C) 2014 V. I. Loganina. This is an open access article distributed under the Creative Commons Attribution License, which permits unrestricted use, distribution, and reproduction in any medium, provided the original work is properly cited.
\end{abstract}

\begin{abstract}
The data on the effect of measurement error on the probability of making a decision on product acceptance at the statistical instability of the production process. It is shown by the example of manufacture of a brick that the probability of a mistake of the first sort grows at increase mean square deviation.
\end{abstract}

Keywords: probability, control, reliability, error of the first kind, the statistics

\section{Introduction}

The quality of construction products and are determined, among other factors, the stability and reproducibility of the manufacturing method. In [1-3] provides information about tuning technology manufacturing processes of concrete products.

Monitoring and measurement are an integral part of process of manufacture of building materials and products. If to designate the valid value of controllable parameter through $U_{v}$, and limiting values as $U_{u . t}$ - the greatest admitted value of parameter and $U_{\text {l.t. }}$ - the least admitted value, a condition of the validity of object of the control (product):

$$
U_{l . t .} \leq U_{v} \leq U_{u . t}
$$

This means that good products are those whose actual values are controlled by the 
parameters specified in the technical specifications, lie between the lowest and highest permissible values. In practice, however, all accompanied by measurement errors caused by the acceptance as a number of good products on the actual deviations beyond the specification limit (probability $\alpha_{i}$ ), and the erroneous reject some part of acceptable products, the actual value of the controlled parameter lie in the tolerance (with probability $\beta_{i}$ ). Deviations result sorting occur within the maximum permissible error of measurement $\pm y_{i}$.

Thus, the objective of quality control of the product depends on the reliability of the measurement results, and, consequently, the accuracy of measuring instruments (MI) [4]. Measurement error y can cause distortion of the actual value of the controlled parameter, that is, result of measurement $U$, containing the random error of measurement y near specification limits on the value of the product, leading to uncertainty estimation of the actual value of the controlled parameter, making it impossible to estimate the real picture: whether the monitored parameter in the tolerance limits or not.

Thus, the necessary condition of the measuring control is to establish a maximum permissible values of the controlled parameter (the tolerance) and measurement error (in this case the error in the MI units of the measured quantity $U$ ). On the basis of the measurement result $y$ from taking a decision on the validity of the controlled product. Besides reliability of the control will be determined and also setting up production process, that is, its statistical stability and reproducibility/

In any form and method of measuring the effectiveness of the control of each object of applied solutions based on the results of measurements $(y)$ is due to the following set of random events that make up a complete group of events:

- The likelihood that a controlled product in a particular parameter fit and is found fit;

- The likelihood that specific parameter controlled by the product is defective and is found to be defective;

- The likelihood that the controlled product uni, but will mistakenly found to be defective - type I error (or false marriage) $(\alpha)$;

- The likelihood that the controlled product is defective, but will wrongly found fit - type II error (or hidden marriage) $(\beta)$.

For finding-out of influence of an error of measurement $y$, and also statistical instability of manufacture on results of grading of products on controllable parameter it is accepted, that laws of dispersion of the valid values of controllable parameter of products and errors of measurements are known. We accept:

1) The law of distribution of parameters of quality;

2) The regular error of manufacturing is equal to zero;

3) The field of the admission of product $\delta$, is limited $U_{\text {l.t. }}$ and $U_{\text {u.t. }}$, and the middle of a field of the admission (rating value) $U_{r}$ can to not coincide with average value of a parameter of quality;

4) The error of measurement $y$ also submits to the normal law with average 
quadratic deviation $\{$ rejection $\} \sigma_{y}$, has no regular component and is characterized by practically limiting value $\pm y_{l}$ ).

The model of the measured $i$ parameter of $n$-parametrical object of the control looks like [5]

$$
z_{i}(t)=U_{i}(t)+y_{i}(t)
$$

where $U_{i}(t)$-true value of the measured parameter;

$y_{i}(t)$ - An error that depends on the time and the probability distribution with density $f\left(U_{i}\right)$ and $f\left(y_{i}\right)$ standard deviation, $\sigma_{U i}$ and $\sigma_{y i}$, respectively.

The joint probability density for the model (1) is given by

$$
f\left(U_{i}, y_{i}\right)=f\left(U_{i}\right) f\left(y_{i} \mid U_{i}\right)
$$

where $f\left(U_{i}\right)$ - the a priori distribution of the measured parameter;

$$
f\left(y_{i} \mid U_{i}\right) \text { - Conditional probability density of error } y_{i} \text { for a given signal. }
$$

Formulas for calculating the risk of the manufacturer $\left(\alpha_{i}\right)$ and consumer's risk $\left(\beta_{i}\right)$ are of the form:

$$
\begin{aligned}
& \alpha_{i}=\int_{a}^{b} f\left(U_{i}\right)\left[\int_{-\infty}^{a_{1}} f\left(y_{i} \mid U_{i}\right) d y_{i}+\int_{b_{1}}^{\infty} f\left(y_{i} \mid U_{i}\right) d y_{i}\right] d U_{i}, \\
& \beta_{i}=\int_{-\infty}^{a} f\left(U_{i}\right)\left[\int_{a_{1}}^{b_{1}} f\left(y_{i} \mid U_{i}\right) d y_{i}\right] d U_{i}+\int_{b}^{\infty} f\left(U_{i}\right)\left[\int_{a_{1}}^{b_{1}} f\left(y_{i} \mid U_{i}\right) d y_{i}\right] d U_{i},
\end{aligned}
$$

where $\left[a_{1} ; b_{1}\right]$ - interval for error $y_{i}$, with the proviso that the values $U_{i}$ fall within the range $[a ; b]$;

$i$ - estimated parameter.

\section{The Results of Studies}

Consider the process of production of ceramic bricks mark 100 as one of the most common types of building materials. Upper tolerance is $U_{u . t}=125 \mathrm{kgs} /$ $\mathrm{cm}^{2}$, lower tolerance $U_{\text {l.t. }}-100 \mathrm{kgs} / \mathrm{cm}^{2}$.

Consider the 3 variants. 
1 variants. By the results of the sample mean value of compressive strength does not coincide with the center of the tolerance field and makes $U_{v}=111 \mathrm{kgs} / \mathrm{cm} 2$, and the standard deviation $\sigma=5,3 \mathrm{kgs} / \mathrm{cm} 2$, the measurement error of $1 \%$.

2 variant. The average value of the compressive strength does not coincide with the center of the tolerance field and makes $U_{v}=108 \mathrm{kgs} / \mathrm{cm}^{2}$, and the standard deviation $\sigma=6,2 \mathrm{kgs} / \mathrm{cm}^{2}$, the measurement error of $1 \%$.

3 variant. The average value of compressive strength coincides with the middle of the tolerance and makes $U_{v}=112,5 \mathrm{kgs} / \mathrm{cm}^{2}$, and the standard deviation $\sigma=$ $4,1 \mathrm{kgs} / \mathrm{cm}^{2}$, the measurement error of $1 \%$.

Results of calculation are shown below.

\section{For variant 1}

$$
\begin{gathered}
\alpha_{1}=\int_{100}^{125} f\left(U_{1}\right)\left[\int_{-\infty}^{1} f\left(y_{1}\right) d y_{1}+\int_{1,25}^{\infty} f\left(y_{1}\right) d y_{1}\right] d U_{1}= \\
=\int_{100}^{125} f\left(U_{1}\right)\left[\left.\lim _{a \rightarrow-\infty} \Phi\left(y_{1}\right)\right|_{a} ^{1}+\lim _{b \rightarrow \infty} \Phi\left(y_{1}\right)_{1,25}^{b}\right] d U_{1}= \\
\int_{100}^{125} f\left(U_{1}\right)\left[\lim _{a \rightarrow-\infty} \Phi\left(\frac{1-1,11}{0,042}\right)-\Phi\left(\frac{a-1,11}{0,042}\right)+\lim _{b \rightarrow-\infty} \Phi\left(\frac{b-1,11}{0,042}\right)-\Phi\left(\frac{1,25-1,11}{0,042}\right)\right] d U_{1}= \\
=\int_{100}^{125} f\left(U_{1}\right)[-\Phi(2,62)+0,5+0,5-\Phi(3,33)] d U_{1}=0,005 \int_{100}^{125} f\left(U_{1}\right) d U_{1}= \\
=0,005\left(\Phi\left(\frac{125-111}{5,3}\right)-\Phi\left(\frac{100-111}{5,3}\right)\right)=0,005(0,4959+0,4812)=0,0049 \\
\beta_{1}=\int_{-\infty}^{100} f\left(U_{1}\right)\left[\int_{1}^{1,25} f\left(y_{1}\right) d y_{1}\right] d U_{1}+\int_{125}^{\infty} f\left(U_{1}\right)\left[\int_{1}^{1,25} f\left(y_{1}\right) d y_{1}\right] d U_{1}= \\
=\int_{-\infty}^{100} f\left(U_{1}\right)\left[\Phi\left(y_{1}\right) \mid 1,25\right] d U_{1}+\int_{125}^{\infty} f\left(U_{1}\right)\left[\Phi\left(y_{1}\right) \mid 1,25\right] d U_{1}= \\
=\int_{-\infty}^{100} f\left(U_{1}\right)\left[\Phi\left(\frac{1,25-1,11}{0,042}\right)-\Phi\left(\frac{1-1,11}{0,042}\right)\right] d U_{1}+\int_{125}^{\infty} f\left(U_{1}\right)\left[\Phi\left(\frac{1,25-1,11}{0,042}\right)-\Phi\left(\frac{1-1,11}{0,042}\right)\right] d U_{1}= \\
=\int_{-\infty}^{100} f\left(U_{1}\right)[0,4994+0,4956] d U_{1}+\int_{125}^{\infty} f\left(U_{1}\right)[0,4994+0,4956] d U_{1}= \\
=0,995\left(\lim _{a \rightarrow-\infty} \Phi\left(\frac{100-111}{5,3}\right)-\Phi\left(\frac{a-111}{5,3}\right)+\lim _{b \rightarrow \infty} \Phi\left(\frac{b-111}{5,3}\right)-\Phi\left(\frac{125-111}{5,3}\right)\right)= \\
=0,995\left(\Phi\left(\frac{100-111}{5,3}\right)+0,5+0,5-\Phi\left(\frac{125-111}{5,3}\right)\right)=0,02279
\end{gathered}
$$




\section{For a variant 2}

$$
\begin{aligned}
& \alpha_{2}=\int_{100}^{125} f\left(U_{2}\right)\left[\int_{-\infty}^{1} f\left(y_{2}\right) d y_{2}+\int_{1,25}^{\infty} f\left(y_{2}\right) d y_{2}\right] d U_{2}= \\
& =\int_{100}^{125} f\left(U_{2}\right)\left[\left.\lim _{a \rightarrow-\infty} \Phi\left(y_{2}\right)\right|_{a} ^{1}+\left.\lim _{b \rightarrow \infty} \Phi\left(y_{2}\right)\right|_{1,25} ^{b}\right] d U_{2}= \\
& =\int_{100}^{125} f\left(U_{2}\right)\left[\lim _{a \rightarrow-\infty} \Phi\left(\frac{1-1,08}{0,042}\right)-\Phi\left(\frac{a-1,08}{0,042}\right)+\lim _{b \rightarrow-\infty} \Phi\left(\frac{b-1,08}{0,042}\right)-\Phi\left(\frac{1,25-1,08}{0,042}\right)\right] d U_{2}= \\
& =\int_{100}^{125} f\left(U_{2}\right)[-\Phi(1,9)+0,5+0,5-\Phi(4)] d U_{2}=0,0288 \int_{100}^{125} f\left(U_{2}\right) d U_{2}= \\
& =0,0288\left(\Phi\left(\frac{125-108}{5,3}\right)-\Phi\left(\frac{100-108}{5,3}\right)\right)=0,0288(0,4969+0,4015)=0,0259 \\
& \beta_{2}=\int_{-\infty}^{100} f\left(U_{2}\right)\left[\int_{1}^{1,25} f\left(y_{2}\right) d y_{2}\right] d U_{2}+\int_{125}^{\infty} f\left(U_{2}\right)\left[\int_{1}^{1,25} f\left(y_{2}\right) d y_{2}\right] d U_{2}= \\
& =\int_{-\infty}^{100} f\left(U_{2}\right)\left[\left.\Phi\left(y_{2}\right)\right|_{1} ^{1,25}\right] d U_{2}+\int_{125}^{\infty} f\left(U_{2}\right)\left[\left.\Phi\left(y_{2}\right)\right|_{1} ^{1,25}\right] d U_{2}= \\
& =\int_{-\infty}^{100} f\left(U_{2}\right)\left[\Phi\left(\frac{1,25-1,08}{0,042}\right)-\Phi\left(\frac{1-1,08}{0,042}\right)\right] d U_{2}+\int_{125}^{\infty} f\left(U_{2}\right)\left[\Phi\left(\frac{1,25-1,08}{0,042}\right)-\Phi\left(\frac{1-1,08}{0,042}\right)\right] d U_{2}= \\
& =\int_{-\infty}^{100} f\left(U_{1}\right)[0,4999+0,4713] d U_{1}+\int_{125}^{\infty} f\left(U_{1}\right)[0,4999+0,4713] d U_{1}= \\
& =0,9712\left(\lim _{a \rightarrow-\infty} \Phi\left(\frac{100-108}{6,2}\right)-\Phi\left(\frac{a-108}{6,2}\right)+\lim _{b \rightarrow \infty} \Phi\left(\frac{b-108}{6,2}\right)-\Phi\left(\frac{125-108}{6,2}\right)\right)= \\
& =0,9712(-\Phi(1,29)+0,5+0,5-\Phi(2,74))=0,0987
\end{aligned}
$$

\section{For a variant 3}

$$
\begin{aligned}
& \alpha_{3}=\int_{100}^{125} f\left(U_{3}\right)\left[\int_{-\infty}^{1} f\left(y_{3}\right) d y_{3}+\int_{1,25}^{\infty} f\left(y_{3}\right) d y_{3}\right] d U_{3}= \\
& =\int_{100}^{125} f\left(U_{3}\right)\left[\left.\lim _{a \rightarrow-\infty} \Phi\left(y_{3}\right)\right|_{a} ^{1}+\left.\lim _{b \rightarrow \infty} \Phi\left(y_{3}\right)\right|_{1,25} ^{b}\right] d U_{3}= \\
& \int_{100}^{125} f\left(U_{3}\right)\left[\lim _{a \rightarrow-\infty} \Phi\left(\frac{1-1,125}{0,042}\right)-\Phi\left(\frac{a-1,125}{0,042}\right)+\lim _{b \rightarrow-\infty} \Phi\left(\frac{b-1,125}{0,042}\right)-\Phi\left(\frac{1,25-1,125}{0,042}\right)\right] d U_{3}= \\
& =\int_{100}^{125} f\left(U_{3}\right)[-\Phi(2,98)+0,5+0,5-\Phi(2,98)] d U_{3}=0,0028 \int_{100}^{125} f\left(U_{3}\right) d U_{3}= \\
& =0,0028\left(\Phi\left(\frac{125-112,5}{4,1}\right)-\Phi\left(\frac{100-112,5}{4,1}\right)\right)=0,0028
\end{aligned}
$$




$$
\begin{aligned}
\beta_{3}=\int_{-\infty}^{100} f\left(U_{3}\right)\left[\int_{1}^{1,25} f\left(y_{3}\right) d y_{3}\right] d U_{3}+\int_{125}^{\infty} f\left(U_{3}\right)\left[\int_{1}^{1,25} f\left(y_{3}\right) d y_{3}\right] d U_{3}= \\
=\int_{-\infty}^{100} f\left(U_{3}\right)\left[\left.\Phi\left(y_{3}\right)\right|_{1} ^{1,25}\right] d U_{3}+\int_{125}^{\infty} f\left(U_{3}\right)\left[\left.\Phi\left(y_{3}\right)\right|_{1} ^{1,25}\right] d U_{3}= \\
=\int_{-\infty}^{100} f\left(U_{3}\right)\left[\Phi\left(\frac{1,25-1,125}{0,042}\right)-\Phi\left(\frac{1-1,125}{0,042}\right)\right] d U_{3}+\int_{125}^{\infty} f\left(U_{3}\right)\left[\Phi\left(\frac{1,25-1,125}{0,042}\right)-\Phi\left(\frac{1-1,125}{0,042}\right)\right] d U_{3}= \\
=\int_{-\infty}^{100} f\left(U_{3}\right)[0,9972] d U_{3}+\int_{125}^{\infty} f\left(U_{3}\right)[0,9972] d U_{3}= \\
=0,9972\left(\lim _{a \rightarrow-\infty} \Phi\left(\frac{100-112,5}{4,1}\right)-\Phi\left(\frac{a-112,5}{4,1}\right)+\lim _{b \rightarrow \infty} \Phi\left(\frac{b-112,5}{4,1}\right)-\Phi\left(\frac{125-112,5}{4,1}\right)\right)= \\
=0,9972(-\Phi(3,05)+0,5+0,5-\Phi(3,05))=0,00269
\end{aligned}
$$

Results of calculation testify, that at increase in value standard deviation increase of probability of occurrence of mistakes I and II sorts is observed. So, for a variant 2 probability of occurrence of mistakes I and II sorts much above also makes accordingly 2,59\% and 9,87\%, while for a variant $1-0,49 \%$ and 2, 279 $\%$. The smallest degree of risks is observed for a variant 3 which is characterized by statistical stability and reproducibility of manufacture. Value probability of occurrence of mistakes I and II sorts make accordingly $0,28 \%$ and 0,269 \%.

\section{Conclusions}

Thus, statistically stable and reproduced process allows to increase reliability of quality assurance of production and to avoid mistakes at decision-making on rejection of production

\section{References}

[1] V. I. Loganina. Organization of statistical acceptance quality assurance of building products and designs. Building materials. 8 (2008), 98 - 99.

[2] V. I. Loganina, B. B. Hrustalev, T. V. Uchaeva. Statistical production management of building products. The East Europe magazine of high technologies 3.t1 (2013), 65 - 67.

[3] V. I. Loganina. To a question on regulation of technological processes of manufacture of concrete of News of higher educational institutions. Construction.3-4 (2009), 42 - 45. 
[4] V. I. Loganina, A. N. Kruglova, V. G. Shuhova, To a question on reliability of the control over manufacture of concrete. The Bulletin of the Belgorod state technological university, 4 (2011), 24 - 26.

[5] V. I. Grey, V.I. Seryh, S.P. Porvatov, V. I. Sedinin, Multi-parameter control of production: reliability and expenses, Methods of Quality Management 5(2010), 48 $-52$.

[6] B. R Levin, Theoretical Foundations of Statistical Radio Engineering. Moscow: Soviet Radio, (1965), 752.

Received: November 12, 2014; Published: December 23, 2014 